\title{
Communication is key: an innovative multidisciplinary approach to communication of regional antimicrobial resistance surveillance data to hospital microbiologists in North East England
}

\author{
SJ Howard \\ From 3rd International Conference on Prevention and Infection Control (ICPIC 2015) \\ Geneva, Switzerland. 16-19 June 2015
}

\section{Introduction}

Antimicrobial resistance (AMR) must be urgently tackled [1]. Strategies to tackle AMR must include using high quality surveillance data to rapidly modify policy and practice. Yet, in the UK and elsewhere, AMR surveillance is conducted by specialists located in organisations separate from hospital practitioners. This leads to communication challenges, including delays, difficulty in communicating technical limitations around interpretation of data, and a lack of clinical interpretation of surveillance data.

\section{Objectives}

This project aimed to improve the quality, speed and clinical relevance of communication between hospital microbiologists and surveillance specialists with regard to AMR surveillance data.

\section{Methods}

A multidisciplinary virtual working group was convened, including hospital microbiologists, the regional microbiologist, a consultant epidemiologist, and a senior information officer, led by a public health specialty registrar. This group developed a short quarterly regional AMR report format, showing and statistically analysing trends, and giving a commentary on the data limitations and clinical significance of the data. The report is thematic, responding to queries and concerns of front-line microbiologists. Discussion of this report at an existing regional microbiology group was encouraged.

North East Health Protection Team, Public Health England, Newcastle upon Tyne, UK 